\title{
Estimación de los servicios ecosistémicos del recurso vegetal de la parroquia Flores, cantón Riobamba
} (c) (i) ()

\section{BY NC SA}

Estimation of the ecosystem services of the vegetable resource of the parroquia Flores, cantón Riobamba

Marcela Yolanda Brito Mancero. ${ }^{1}$ \& Nataly Alexandra Garcés Chérres. ${ }^{2}$

Recibido: 15-06-2021 / Revisado: 25-06-2021 /Aceptado: 13-07-2021/ Publicado: 05-08-2021

\begin{abstract}
.
DOI: https://doi.org/10.33262/concienciadigital.v4i3.1.1817

Introduction. The SE are benefits that nature produces for the welfare of humanity, the plant resource is recognized mainly because it provides food and is related to ancestral knowledge. Objective. Estimate the economic value of the productive plant resource of the parroquia Flores. Methodology. The characteristics of the area, sociodemographic variables around the vegetable resource were qualitatively analyzed, the total income received by the producers (vegetable, forest and pastures) was calculated from the average yields and production costs for the vegetable resource, in order to the forest resource and grasslands were estimated from market prices. The contingent value method was used, 350 people from 4 communities of the parish were surveyed under a hypothetical scenario of conservation of the plant resource, the willingness to pay of the users was estimated. Results. The age variable, for provisioning services, this service was the best scored with a value of 9.2 / 10, this value indicates that users value tangible services more, that generate benefits, the least valued service was that of ancestral practices with a value of $(5,6)$. Regarding the Willingness to pay, most of the users $(85 \%)$, would not be willing to

${ }^{1}$ Escuela Superior Politécnica de Chimborazo, Facultad de Recursos Naturales. Riobamba, Ecuador. mybrito@espoch.edu.ec, https://orcid.org/0000-0003-2689-3516

2 Investigador independiente en el área, alexandrita.garces@gmail.com, https://orcid.org/0000-0001-90638873
\end{abstract}


cancel because they attribute the responsibility for conservation to the government, $15 \%$ are willing to pay $\$ 10.20$ per year. The total environmental economic value of the productive plant resource calculated was $\$ 724,730.63$, a variable value around the time of year or other external. Conclusions. The supply services in the provision of food were the most appreciated, the least valued were the cultural services in ancestral rituals. The sociodemographic variable of age affects the assessment of both supply and cultural services. In the VET, pasture production obtained the best valuation followed by forests and finally agricultural production since this is marginal and poor with little investment and irrigation deficiency.

Keywords: Ecosystem services, environmental services, environmental economy, environmental assessment, parroquia Flores.

\section{Resumen.}

Introducción. Los SE son beneficios que la naturaleza produce para bienestar de la humanidad, el recurso vegetal es reconocido principalmente porque proporciona alimentos y se relacionada con los saberes ancestrales. Objetivo. Estimar el valor económico del recurso vegetal productivo de la parroquia Flores. Metodología. Se analizó cualitativamente las características de la zona, variables sociodemográficas en torno al recurso vegetal, se calculó los ingresos totales que perciben los productores (vegetal, forestal y pastos) a partir de los rendimientos promedio y los costos de producción para el recurso vegetal, para el recurso forestal y pastizales se estimaron partir de precios de mercado. Se utilizó el método de valor contingente, se encuestaron a 350 personas de 4 comunidades de la parroquia bajo un escenario hipotético de conservación del recurso vegetal, se estimó la disposición a pagar de los usuarios. Resultados. La variable edad, para los servicios de aprovisionamiento, este servicio fue el mejor puntuado con un valor de 9,2/10, este valor indica que los usuarios valoran más los servicios tangibles, que les generen beneficios, el servicio menos valorado fue el de prácticas ancestrales con un valor de $(5,6)$. En lo referente a la Disposición a pagar, la mayor parte de los usuarios (85\%), no estarían dispuestos a cancelar pues atribuyen la responsabilidad de la conservación al gobierno el $15 \%$ está dispuesto a pagar $\$ 10,20$ anual. El valor económico ambiental total del recurso vegetal productivo calculado fue $\$ 724.730,63$, valor variable en torno a la época del año u otros factores externos. Conclusiones. Los Servicios de abastecimiento en el aprovisionamiento de alimentos fuero los más apreciados, los de menor valoración fueron los servicios culturales en rituales ancestrales. La variable sociodemográfica de la edad incide en la valoración de los servicios tanto de abastecimiento como culturales. En la VET la producción de pasto obtuvo la mejor valoración seguido por bosques y finalmente la producción agrícola ya que esta es marginal y pobre con poca inversión y deficiencia de riego.

Palabras claves: Servicios ecosistémicos, servicios ambientales, economía ambiental, valoración ambiental, parroquia Flores. 


\section{Introducción.}

La reducción de los servicios ecosistémicos desempeña un papel clave en el grupo de fenómenos que se denomina crisis ecológica global. La explosión demográfica ha resultado en una sobrepoblación de nuestro planeta. (Hufnagel, et al, 2018)

Para Hufnagel (2018), el deterioro de las condiciones ecológicas ha causado problemas sociales directa e indirectamente, como epidemias, pobreza y crisis humanitarias. Además de estas, las actividades económicas contaminantes que destruyen la naturaleza aumentan la riqueza y la desigualdad de ingresos entre las personas, lo que resulta en más tensiones sociales (crimen, terrorismo, disturbios y guerras).

La situación de Ecuador se ha visto amenazada como resultados de más de quinientos años de sobrexplotación de los recursos naturales y excesiva emanación de sustancias contaminantes al ambiente. Es de vital importancia conservar y restaurar los ecosistemas para que provean los bienes y servicios de calidad a las generaciones. (Ministerio del Ambiente-Ecuador, 2016)

La cobertura de suelos indica que el $75,10 \%$ pertenece a bosques secundarios, de la misma manera esta cobertura presenta degradación debido a la quema, y las condiciones económicas de la zona.

La actividad agro productiva de la parroquia Flores presenta complejas relaciones debido a la carencia del sistema riego, dando un lugar a suelos infértiles y erosionados que generan baja producción agropecuaria y conllevan a una baja condición socioeconómica baja. (GADPR- Flores, 2015)

La producción es mayormente orgánica lo que aporta a la soberanía alimentaria, uno de los problemas que enfrenta Flores es que los usuarios prefieren consumir alimentos de afuera como enlatados, harinas, arroz, entre otros. (GADPR- Flores, 2015)

El bajo rendimiento de la producción agrícola se le atribuye a las condiciones climáticas $57,14 \%$ en sequias, el $42,86 \%$ para heladas, además de que en la parroquia predomina el minifundio, dando como resultado una agricultura de subsistencia que condiciona la actividad económica de sus pobladores. (GADPR- Flores, 2015)

Calcular el valor económico ambiental del recurso vegetal podría contribuir positivamente a la sociedad, ya que muestra los costos que serían causados por la pérdida de biodiversidad y la degradación de los ecosistemas de la parroquia a su vez fomenta una gestión más eficiente que permite una mejor conservación y protección de los activos ambientales y evita su degradación. (Estruch, 2018)

\section{Metodología.}

La investigación realizada es de tipo Descriptiva Partiendo de la caracterización de los componentes de la parroquia Flores que inciden en la valoración del recurso vegetal 
productivo, transversal en el año 2020 y correlacional que relaciona variables significativas que tengan relación con los servicios ecosistémicos en estudio.

La población de estudio fue los habitantes de la Parroquia Flores que es de 5548 y las especies vegetales que hay en ella. Para la determinación del tamaño de la muestra se utilizó la fórmula de Canavos (1998), que dio como resultado 325 encuestas realizadas, para la definición de las unidades de estudio se revisó la información disponible del Plan de Ordenamiento territorial parroquial y se estableció 4 comunidades Naubug, Capiatá, Guantual Grande central y Puesetus Grande, según los parámetros de números de habitantes y producción.

En cuanto a la identificación de variables se trabajó con las características sociodemográficas de los habitantes de Flores como variable independiente y variable dependiente la valoración económica de los recursos vegetales de la parroquia Flores.

En cuanto a las técnicas de recolección de datos, para la caracterización de la zona de estudio se utilizó la revisión bibliográfica de documentación del Plan de ordenamiento territorial vigente, datos del instituto nacional de estadísticas y censos (INEC), la técnica de consulta a las partes interesadas identificadas las cuales fueron: representantes del gobierno autónomo descentralizado de la parroquia rural Flores, productores de la parroquia, representantes del ministerio de agricultura y ganadería y representantes de organizaciones de la comunidad. La técnica de encuesta a los pobladores estuvo distribuida en tres secciones: componente social, componente ambiental con el escenario de conservación del recurso vegetal productivo y el componente monetario con la disposición a pagar.

Para la valoración ambiental de los servicios ecosistémicos se utilizó la escala de Likert donde los pobladores entrevistados quienes apreciaron los servicios en una escala de 1 a 10. Para la información acerca del recurso vegetal se utilizó datos de la población económicamente activa (PEA), principales actividades económicas, principales productos, problemática ambiental, bienes y servicios ambientales e información cartográfica de la zona de estudio del Ministerio de agricultura y ganadería.

La técnica para evaluar económica y ambientalmente el recurso vegetal fue mediante la aplicación del método de valor contingente de valoración económica.

Figura 1: Componentes del Valor económico toral (VET)

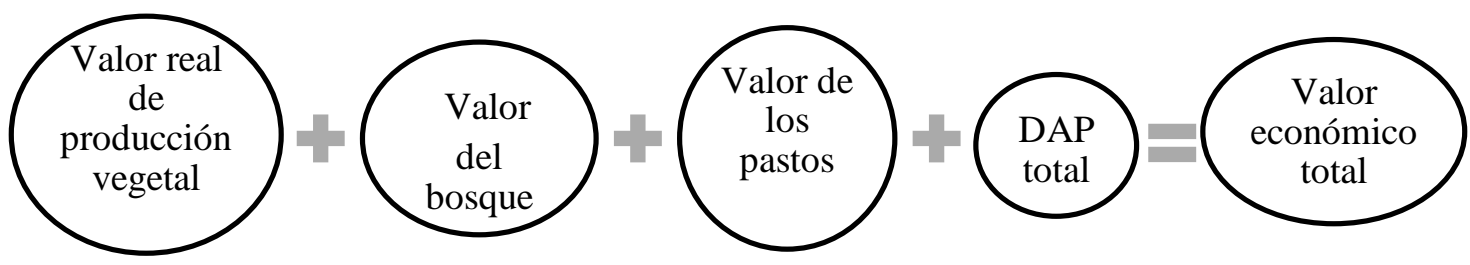

Elaboración: Los autores 
Valor real de producción vegetal para lo cual se realizó la diferencia entre los precios de mercado de los principales recursos vegetales con el costo de producción de los mismos, el valor de bosque que se identificó mediante las licencias forestales y el valor del pasto se identificó directamente con los encuestados en sus precios locales. La disposición a pagar DAP se determinó a través del escenario hipotético de conservación que los usuarios enunciaron.

\section{Resultados.}

\section{Caracterización de la zona de estudio}

Figura 2: Límites de la parroquia Flores

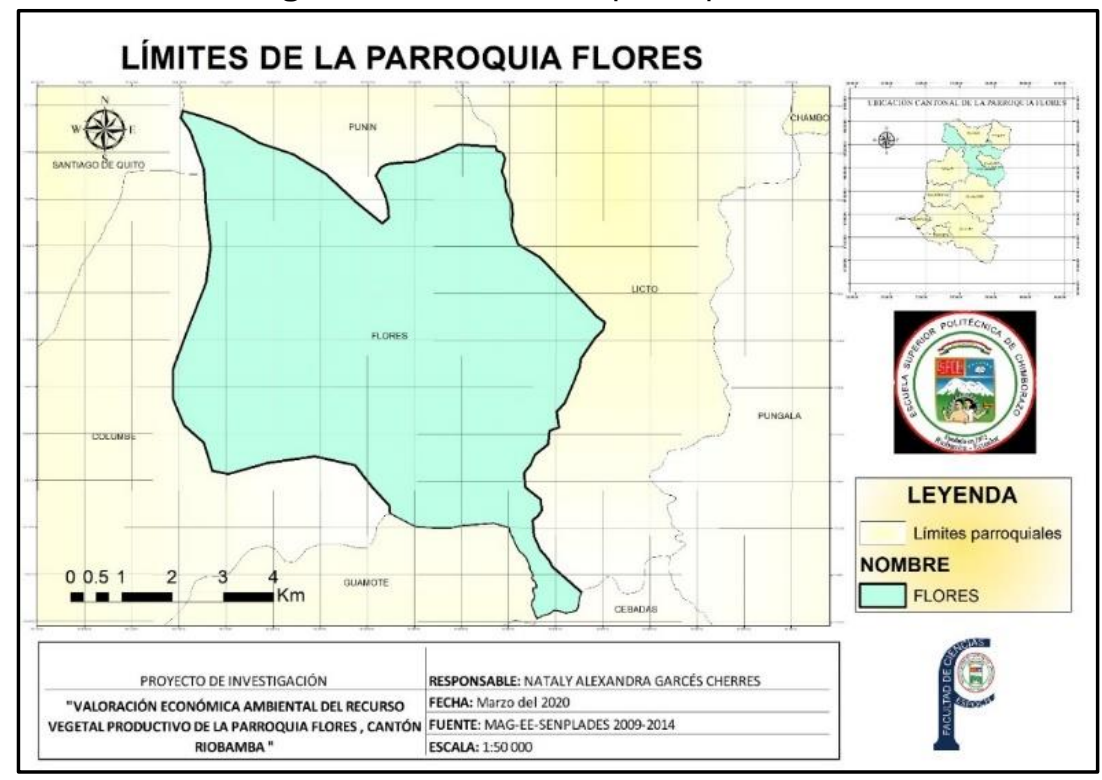

Elaboración: Nataly Garcés (2020)

Ubicación de la parroquia Flores: La parroquia Flores se encuentra ubicada en el cantón Riobamba, Provincia de Chimborazo en una altitud que varía de $2840 \mathrm{~m}-3555 \mathrm{msnm}$, sus límites son: al norte por la parroquia Punín, al sur la matriz de Guamote y Cebadas, al este la parroquia Licto, al oeste las parroquias de Columbe y Punín. Las coordenadas GPS de la parroquia Flores son: latitud: -1.8 y longitud: -78.6333 .

Clima: Flores posee un clima frio con temperaturas que oscilan de $10^{0} \mathrm{C}$ a $16^{0} \mathrm{C}$, y una media de $11^{\circ} \mathrm{C}$, la precipitación se encuentra entre $500 \mathrm{~mm}$ a $1250 \mathrm{~mm}$ al año, se observa la presencia de vientos fuertes en verano sobre todo en el mes de agosto, las heladas se presentan en los meses de mayo, agosto y diciembre; neblinas en abril y sequías en de junio y diciembre. (GADPR- Flores, 2015)

Suelo: Los presentan pendientes suaves de hasta $25 \%$, pendientes fuertes de 40 a $70 \%$ y muy fuertes con más del 70\%. pH que va de 4,5 extremadamente ácido a 7 neutro. Las texturas presentes son variables se encuentra suelos francos, franco limoso, francos 
arcillosos y texturas gruesas. (GADPR- Flores, 2015). En la tabla $\mathrm{N}^{\circ} 01$ se presenta los análisis de suelos de la comunidad de Nabug.

Tabla $\mathbf{N}^{\circ}$ 01: Análisis de suelos Comunidad de Nabug - Parroquia Flores

ANÁLISIS UNIDADES RESULTADOS

\begin{tabular}{ccc}
\hline$p H$ & Unidades de $\mathrm{pH}$ & 5,7 \\
Conductividad & $\mu \mathrm{S} / \mathrm{cm}$ & 101,4 \\
Textura & $\mathrm{N} / \mathrm{A}$ & Franco arcilloso \\
Humedad & $\%$ & 21,55 \\
Materia Orgánica & $\%$ & $3,18 \%$ \\
\hline
\end{tabular}

Fuente: (Garcés, 2021)

Elaboración: Los autores

El uso de suelo en la parroquia se distribuye de la siguiente manera; la mayor parte el $75,10 \%$ se encuentra cubierto por bosque, seguido por pasturas con el $23,20 \%$, el $0,19 \%$ a cuerpos de agua y el $1,51 \%$ corresponde a cultivos.

Figura 3: Mapa de aptitud agrícola de la parroquia Flores

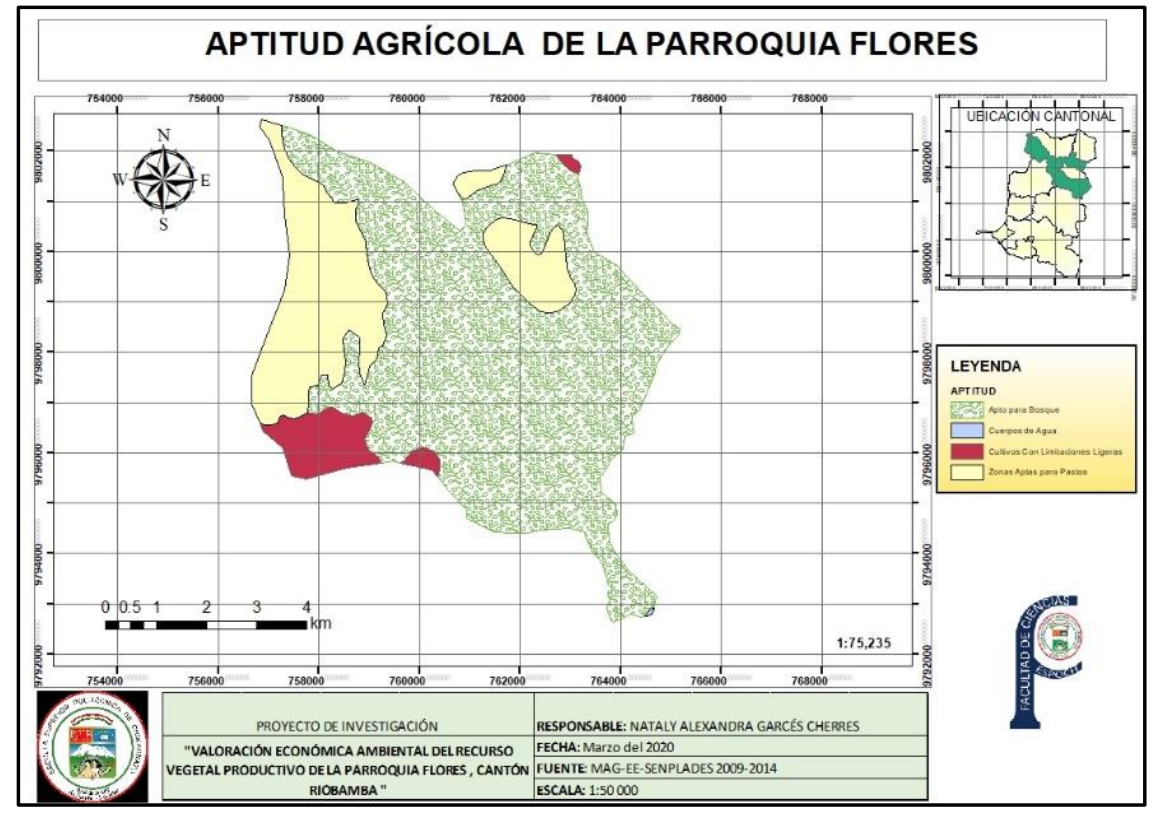

Elaboración: Nataly Garcés (2020)

En cuanto a la aptitud agrícola, en la parroquia Flores se observa que se dedican a los cultivos extensivos, que la mano de obra es escasa, el uso de agua y agroquímicos es mínimo. Los cultivos que prevalecen son gramíneas como la cebada, el maíz sueve seco y quinua, hortalizas como la papa y la cebolla y leguminosas como el haba (Tabla $\mathrm{N}^{\circ} 02$ ), cultivos como fréjol, melloco, oca, mashua, se encuentra formando parte de los mosaicos agropecuarios, es decir no se ha definido espacios dedicados únicamente a estos cultivos; 
las áreas cosechas o con poca productividad son aprovechadas para realizar el pastoreo nómada de ovejas y ganado.

Tabla $N^{\circ}$ 02: Tipos de cultivo de la parroquia Flores

\begin{tabular}{|c|c|c|c|}
\hline \multicolumn{2}{|c|}{ TIPO DE CULTIVO } & \multirow{2}{*}{$\begin{array}{c}\text { SUPERFICIE (ha) } \\
65\end{array}$} & \multirow{2}{*}{$\begin{array}{r}\% \\
31,9\end{array}$} \\
\hline Gramíneas & Maíz suave seco & & \\
\hline & Cebada & 40 & 19,7 \\
\hline & Quinua & 20 & 9,82 \\
\hline \multirow[t]{2}{*}{ Hortalizas } & Papa & 55,9 & 27,5 \\
\hline & Cebolla blanca & 7,1 & 3,47 \\
\hline leguminosas & Haba tierna & 15,6 & 7.66 \\
\hline
\end{tabular}

En lo referente a riego de la parroquia Flores esta se abastece de la subcuenca del Río Chambo, por las particularidades de ubicación geográfica el sistema de riego aquí es deficiente, la superficie total con riego comprende un total $14 \mathrm{Ha}$, siendo el sistema de riego por inundación es la forma de aplicación de riego.

\section{Análisis del componente social.}

De las 325 encuestas aplicadas en los habitantes de las comunidades con mayor número de pobladores Naubug, Capiatá, Guantual Grande central y Puesetus Grande se obtuvo los siguientes resultados sociodemográficos.

Tabla N03: Características sociodemográficas que inciden en la valoración de los servicios ecosistémicos

\begin{tabular}{|c|c|c|}
\hline CARACTERÍSTICAS & VARIABLES & PORCENTAJE \\
\hline \multirow[t]{2}{*}{ GÉNERO } & Hombre & $43,83 \%$ \\
\hline & Mujer & $56,47 \%$ \\
\hline \multirow[t]{5}{*}{ ESTADO CIVIL } & Soltero & $25,30 \%$ \\
\hline & Unión libre & $8,52 \%$ \\
\hline & Casado & $60,10 \%$ \\
\hline & Divorciado & $1,22 \%$ \\
\hline & Viudo & $4,87 \%$ \\
\hline EDAD & Menor a 30 años & $15,00 \%$ \\
\hline
\end{tabular}




\begin{tabular}{|c|c|c|}
\hline & $31-60$ años & $66,00 \%$ \\
\hline & Mayor 60 años & $19,00 \%$ \\
\hline \multirow[t]{7}{*}{ EDUCACIÓN } & Primaria incompleta & $32,50 \%$ \\
\hline & Primaria completa & $37,05 \%$ \\
\hline & Secundaria incompleta & $8,18 \%$ \\
\hline & Secundaria completa & $15,91 \%$ \\
\hline & Tercer nivel incompleto & $3,18 \%$ \\
\hline & Tercer nivel completo & $2,95 \%$ \\
\hline & Cuarto nivel & $0,23 \%$ \\
\hline \multirow[t]{6}{*}{ OCUPACIÓN } & Ama de casa & $19,00 \%$ \\
\hline & Empleado & $6,91 \%$ \\
\hline & Independiente & $56,00 \%$ \\
\hline & desempleado & $9,00 \%$ \\
\hline & Estudiante & $9,63 \%$ \\
\hline & Jubilado & $0,00 \%$ \\
\hline \multirow[t]{5}{*}{ INGRESOS } & Menor a 394 dólares & $87,35 \%$ \\
\hline & 494 - 778 dólares & $6,17 \%$ \\
\hline & 778 a 1000 dólares & $4,63 \%$ \\
\hline & 1000 a 2000 dólares & $1,54 \%$ \\
\hline & mayor a 2000 dólares & $31,00 \%$ \\
\hline
\end{tabular}

Fuente: (Garcés, 2021)

Elaboración: Los autores

Entre los datos más representativos en el análisis sociodemográfico se encuentra que en el componente edad donde la población joven menor a 30 años representa el valor más bajo con el $15 \%$ lo que coincide con lo establecido por GADM - Riobamba en su documento de línea base de grupos de atención prioritaria del cantón Riobamba donde manifiestan que Flores que en la población joven en edad de trabajar han abandonado la parroquia (Consejo Cantonal de Protección de derechos GADM Riobamba, 2019). En cuanto al nivel de instrucción se manifiesta que el 69,55\% de personas encuestadas 
cursaron la primaria de forma completa o incompleta, en cuanto a ocupación se obtiene que el $56 \%$ de la población se ocupa en actividades como independientes siendo la agricultura y el pastoreo las principales actividades, y en lo referente a los ingresos se obtuvo que el $87,53 \%$ de los encuestados ganan menos del salario mínimo del Ecuador

\section{Análisis del componente ambiental}

El componente ambiental se analizó usando el escenario de conservación del recurso vegetal productivo, mediante la identificación y valoración de los servicios ambientales donde los encuestados apreciaron cada servicio utilizando la escala de Likert de 1 a 10 donde 1 correspondía a no importante y 10 los resultados se observan en la tabla $\mathrm{N}^{\circ} 04$

Tabla N04: Valoración ambiental de los servicios ecosistémicos de la parroquia Flores

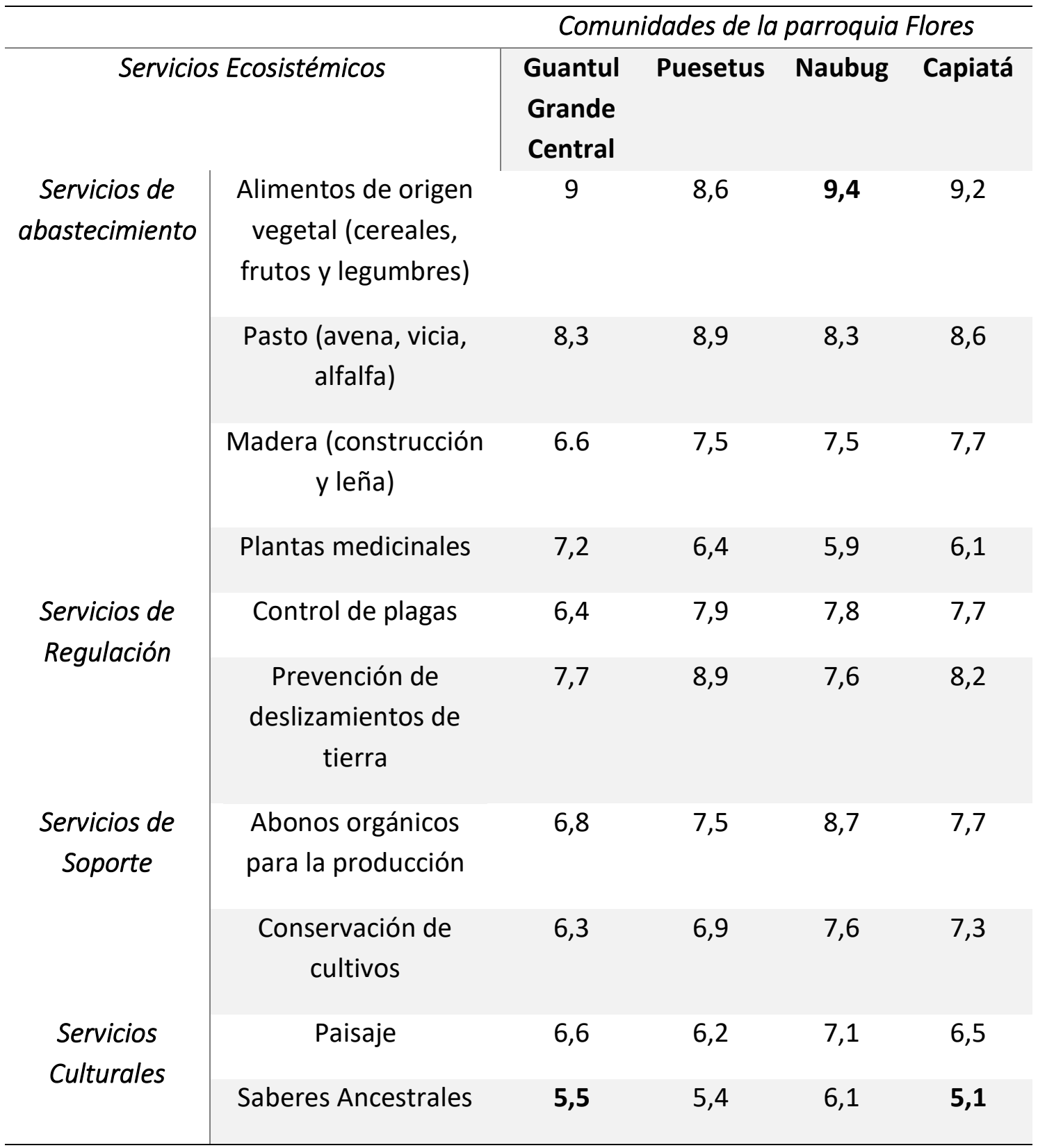

Fuente: (Garcés, 2021)

Elaboración: Los autores 
Los servicios ambientales mejores puntuados (Tabla $\mathrm{N}^{\circ} 04$ y Tabla $\mathrm{N}^{\circ} 05$ ) son los servicios de abastecimiento con un valor medio de 8,5 siendo los pobladores de la comunidad de Nabug quienes mejores valoraron este servicio en el componente de producción de alimentos llegando a 9,4 lo que está relacionado con lo que mencionan el el plan de ordenamiento territorial en que estas comunidades poseen cultivos para la comercialización y para el autoconsumo (GADPR- Flores, 2015). Dos servicios presentan valores muy cercanos entre sí, los servicios de regulación con 7,7 se encuentran ligeramente por encima de los servicios de soporte con 7,4 esta pequeña diferencia se le puede atribuir a que los servicios de soporte son más complejos de percibir (Agbenyega et al, 2019) pero se encuentran sobre los valores que obtuvieron los servicios culturales por lo que se considera que estos ocupan el segundo lugar en las preferencias de los encuestados, siendo el control de plagas es el mejor valorado. Mientras que los servicios culturales muestran el valor medio más bajo con 6 siendo la comunidad de Capiata quienes dieron los presentaron los valores más bajos siendo los saberes ancestrales los menor puntuados con 5,1 lo que concuerda con lo mencionado por la FAO quienes enuncian que los conocimientos ancestrales e indicadores culturales han ido mermando en las comunidades indígenas y a medida que pasan los años pueden desaparecer. (FAOBolivia, 2013).

La jerarquización realizada por los habitantes de la parroquia rural Flores se ajusta a lo mencionado en trabajos previos realizado en áreas rurales donde se evidencia que las preferencias de los servicios ambientales se encuentran en el siguiente orden servicios de abastecimiento, seguidos por los de regulación y finalmente los culturales. (Villamagua, 2017)

Tabla $\mathbf{N}^{\circ}$ 05: Promedio por grupo de los servicios ambientales

\begin{tabular}{|c|c|c|c|c|c|c|c|c|c|c|}
\hline SERVICIOS & \multicolumn{4}{|c|}{ Abastecimiento } & \multicolumn{2}{|c|}{ Regulación } & \multicolumn{2}{|c|}{ Soporte } & \multicolumn{2}{|c|}{ Culturales } \\
\hline $\begin{array}{c}\text { AMBIENTALE } \\
S\end{array}$ & 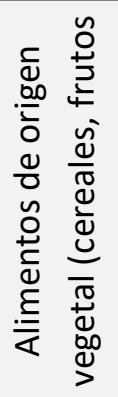 & $\begin{array}{l}\frac{\pi}{\frac{\pi}{\pi}} \\
\frac{0}{\pi} \\
\frac{\pi}{\pi} \\
\frac{\pi}{\pi} \\
\frac{\Phi}{\pi} \\
\frac{\pi}{\pi} \\
\frac{\pi}{\pi} \\
0 \\
\frac{\pi}{\pi} \\
\frac{\pi}{0}\end{array}$ & 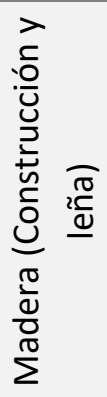 & 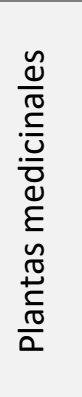 & 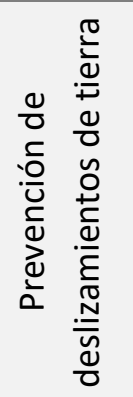 & $\begin{array}{l}0 \\
0 \\
0 \\
\frac{0}{0} \\
0 \\
0 \\
0 \\
0 \\
0 \\
0 \\
0 \\
0 \\
0\end{array}$ & 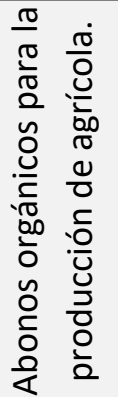 & 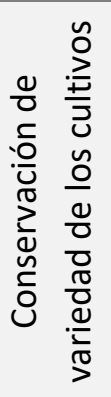 & $\frac{\frac{0}{\pi}}{\frac{\pi}{\pi}}$ & 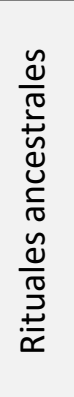 \\
\hline $\begin{array}{c}\text { Valor medio } \\
\text { por } \\
\text { componente } \\
\text { de servicio }\end{array}$ & 9,2 & 8,6 & 7,8 & 6,5 & 7,5 & 7,7 & 6,7 & 7,2 & 6,6 & 5,6 \\
\hline $\begin{array}{l}\text { Valor medio } \\
\text { del servicio } \\
\text { ambiental }\end{array}$ & & 8, & & & 7,7 & & 7, & & & \\
\hline
\end{tabular}

Fuente: (Garcés, 2021)

Elaboración: Los autores 
En lo referente a la relación de las variables sociodemográficas en la valoración de los servicios ambientales (Tabla $\mathrm{N}^{\circ} 06$ ) se obtuvo que la variable edad incide en la valoración de los servicios de abastecimiento concordando con lo establecido por (Briceño, 2016) que señala que la población adulta les atribuye más importancia a los servicios de aprovisionamiento.

La variable edad además tiene incidencia en la percepción del servicio culturales, el grupo de personas mayores a 50 años estiman valores más a su entorno esto puede deberse a como indica (INTA, 2015) el tiempo de interacción continua de los seres humanos con los recursos naturales les permite valorarlos de mejor modo y así determinar la identidad cultural y el sentido de pertenencia de una comunidad (Leiva, 2018).

Tabla N06: Variables que inciden en la valoración de los servicios ecosistémicos

\begin{tabular}{|c|c|c|c|c|c|c|}
\hline \multirow[b]{2}{*}{$\begin{array}{l}\text { VARIABLES SOCIO } \\
\text { DEMOGRÁFICAS }\end{array}$} & \multirow[b]{2}{*}{$\begin{array}{l}\text { PRUEBA T Y NO } \\
\text { PARAMÉTRICA }\end{array}$} & \multirow[b]{2}{*}{$\begin{array}{l}\text { ANNOVA DE } \\
\text { UN FACTOR }\end{array}$} & \multicolumn{4}{|c|}{ VALOR $p$} \\
\hline & & & SA & SR & SS & SC \\
\hline Sexo & $x$ & & 0,87 & 0,075 & 0,244 & 0,140 \\
\hline Edad & & $x$ & 0,003 & 0,323 & 0,282 & $5,67 E-11$ \\
\hline Estado civil & $x$ & & 0,36 & 0,54 & 0,75 & 0,59 \\
\hline Nivel de educación & & $x$ & 0,002 & 0,221 & 0,853 & 0,35 \\
\hline Ingresos mensuales & & $x$ & 0,32 & 0,53 & $4,25 E-5$ & 0,98 \\
\hline Ocupación & & $x$ & 0,98 & 0,06 & 0,84 & 0,43 \\
\hline
\end{tabular}

Fuente: (Garcés, 2021)

Elaboración: Los autores

\section{Análisis del componente monetario}

\section{Producción agrícola}

En el análisis de la tabla $\mathrm{N}^{\circ} 07$, la parroquia Flores presenta una baja producción agropecuaria según lo refiere (GADPR- Flores, 2015), los sistemas productivos son marginales si los productores mejoran la cantidad y calidad de riego, adquieren tecnificación y hacen uso de una mayor y mejor cantidad de fertilizantes en un cultivo se podrá obtener mayor producción, pero la incorrecta utilización de la tecnificación y fertilización podría llegar a afectar de manera negativa el nivel y calidad del producto. (VIU, 2018)

Otro factor que incide en la baja productividad es la carencia de servicios de asistencia técnica y créditos, lo cual propicia a los pobladores a migrar hacia la ciudad y otros países. (Consejo Cantonal de Protección de derechos GADM Riobamba, 2019). 
En la tabla $\mathrm{N}^{\circ} 08$, se establece los cálculos de las especies forestales siendo el Pinus radiata y el Eucalyptus globulus las especies predominantes en la parroquia rural Flores lo que coincide con lo mencionado por (Paltan, 2020) \& (Guevara, 2020)

Tabla N07: Cálculo de producción de cultivos predominantes

\begin{tabular}{|c|c|c|c|c|c|c|c|}
\hline \multirow[t]{2}{*}{ Cultivo } & 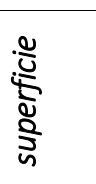 & 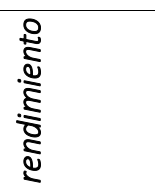 & 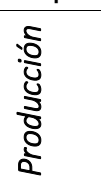 & 芯 & 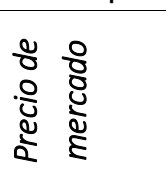 & 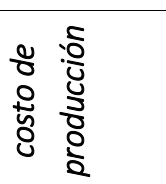 & 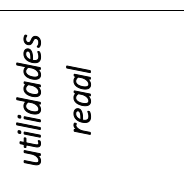 \\
\hline & (Ha) & (Ton/Ha) & (Ton) & (\$)/Ton & (\$) & (\$) & (\$) \\
\hline Maíz suave seco & 65 & 0,76 & 49,4 & 400 & $19.760,00$ & & $-6.100,00$ \\
\hline Papa & 55,9 & 0,78 & 43,6 & 560 & $24.417,12$ & $21.975,48$ & $2.441,64$ \\
\hline Cebada & 40 & 0,89 & 35,6 & 600 & $21.360,00$ & $14.311,20$ & $7.048,80$ \\
\hline Quinua & 20 & 0,65 & 13 & $1.900,00$ & $24.700,00$ & $18.525,35$ & $6.174,65$ \\
\hline Haba tierna & 15,6 & 0,55 & 8,58 & 140 & $1.201,20$ & $1.057,23$ & 143,97 \\
\hline Cebolla blanca & 7,1 & 0,35 & 2,485 & 525 & $1.304,63$ & $1.023,87$ & 280,76 \\
\hline Total & & & & & $92.742,95$ & $82.753,13$ & $\$ 22.189,82$ \\
\hline
\end{tabular}

Fuente: (Garcés, 2021)

Elaboración: Los autores

Producción Forestal

Tabla N08: Valor de la producción Forestal

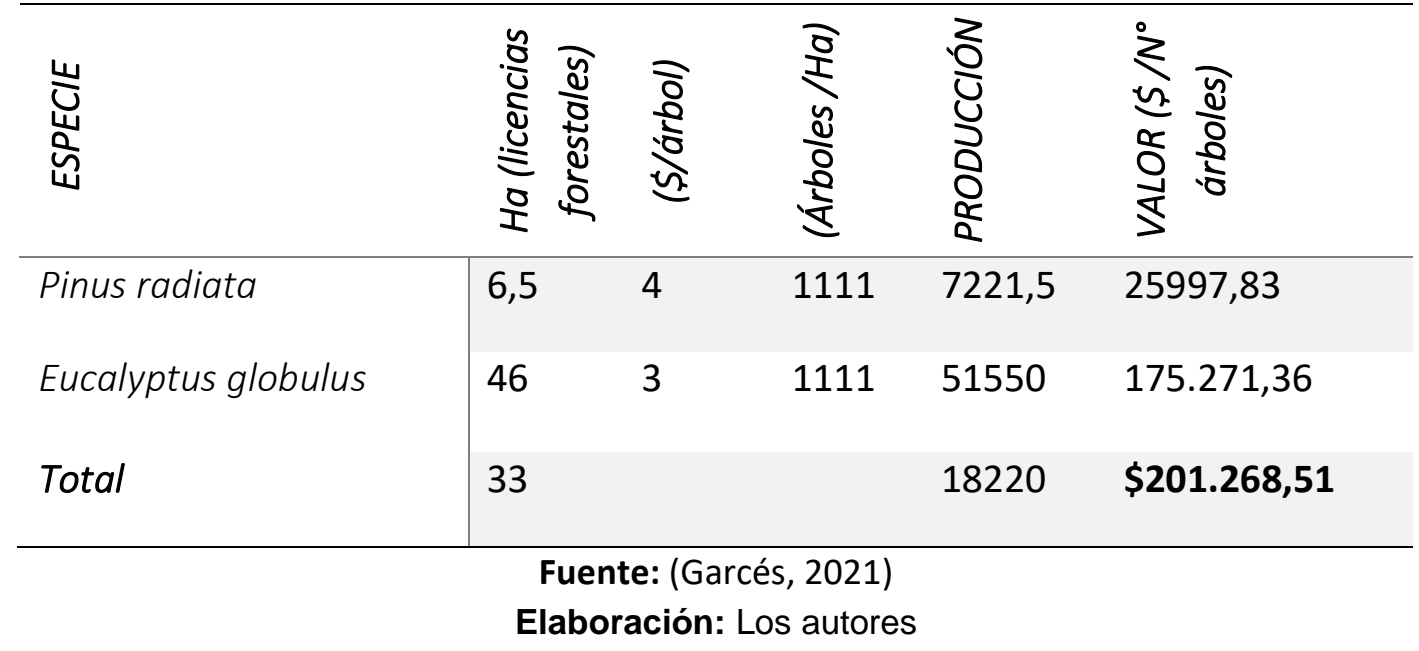

Producción de pastizales

Tabla $\mathbf{N}^{\circ} 09$ : Valor de producción de pastizales

\begin{tabular}{c|ccc}
\hline & Cobertura $(\mathrm{Ha})$ & Precio $\$ /$ ha & Valor total \\
\hline Pastos & $\mathbf{8 2 5 , 5}$ & 565 & $\mathbf{\$ 4 6 6 . 4 0 7 , 5}$ \\
\hline
\end{tabular}

Fuente: (GADPR- Flores, 2015)

Elaboración: Los autores 


\section{Disposicion a pagar}

En las Gráficas 01 y 02 se observa que la mayor parte de la población encuestada el $85 \%$ no está dispuesto a pagar para la conservación de los servicios ecosistémicos que provee el recurso vegetal productivo por diversos motivos entre ellos el más sobresaliente está en que le atribuyen al gobierno la responsabilidad de conservar el recurso vegetal lo que no concuerda con las razones expuestas en estudios anteriores donde se evidencia que la principal razón por la que la población no está dispuesta a pagar tiene que ver con que el nivel de ingresos no llega al salario mínimo vital (Lemache, 2020) el 15\% restante pagaría hasta un valor del $\$ 10,20$ anual.

Grafica N 01: DAP de los usuarios de la parroquia

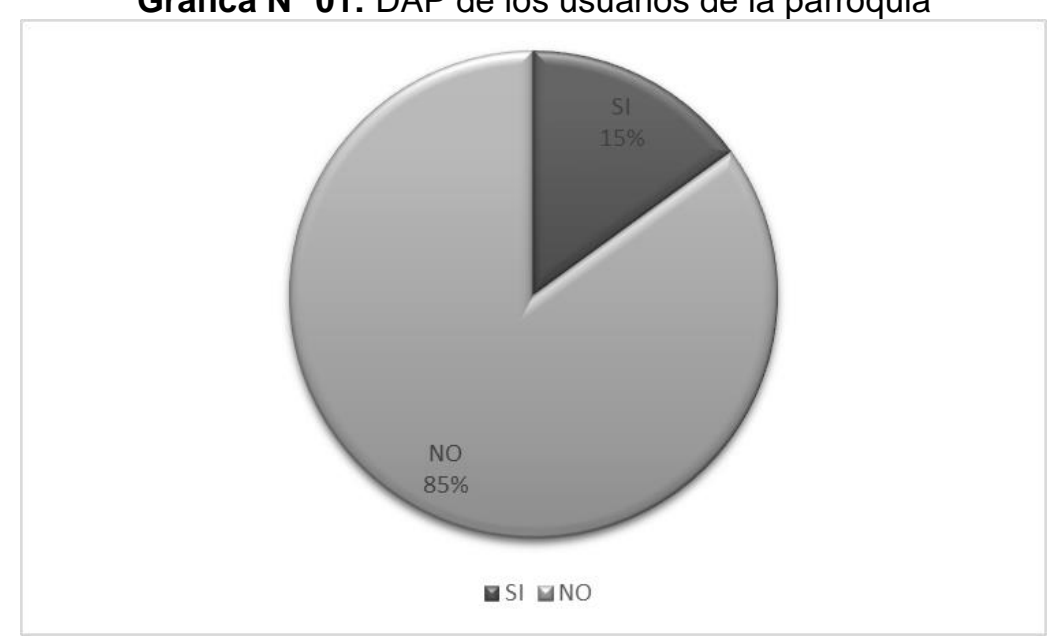

Fuente: (Garcés, 2021)

Elaboración: Los autores

Grafica N 02: Motivos por lo que los usuarios no estarian dispuestos a pagar

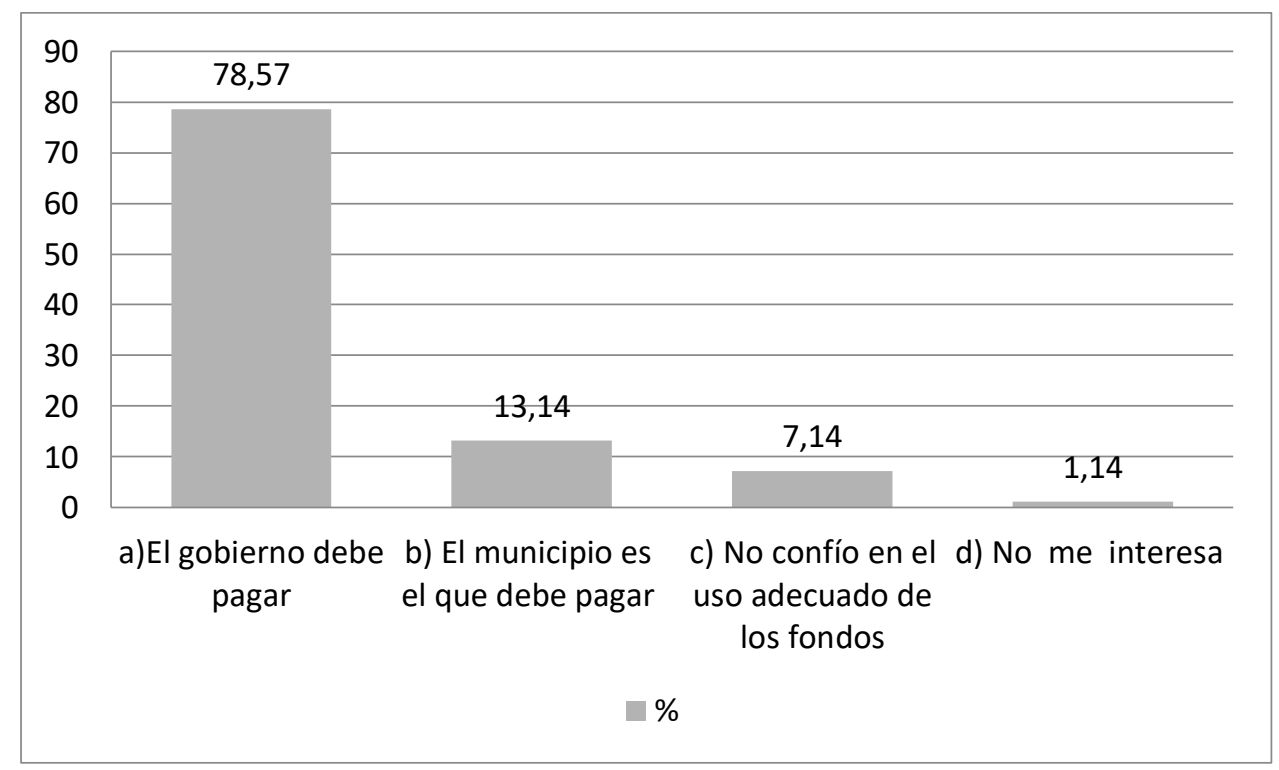

Fuente: (Garcés, 2021)

Elaboración: Los autores 
Tabla 10: DAP total anual

$D A P(\$ / a \tilde{n} o)$

$\$ 10,20$
PEA (2020)

3419

$\$ 34.873,8$

Fuente: (Garcés, 2021)

Elaboración: Los autores

Valor económico ambiental total del recurso vegetal productivo

Tabla N`11: Cálculo del VET del recurso vegetal productivo

\begin{tabular}{c|cr}
\hline \multicolumn{1}{c}{ TIPO DE VALOR } & COMPONENTE & VALOR MONETARIO (\$) \\
\hline Valor de uso directo & Recurso vegetal & $\$ 22.189,82$ \\
Valor de uso directo & Bosques & $\$ 201.268,51$ \\
Valor de uso directo & Pastos & $\$ 466.407,50$ \\
DAP & & $\$ 34.873,80$ \\
VET total & & $\$ 724.730,63$
\end{tabular}

Fuente: (Garcés, 2021)

Elaboración: Los autores

El valor monetario total calculado para el recurso vegetal productivo de la parroquia Flores se estimó en $\$ 689.865,83$, dicho valor puede variar de acuerdo a los rendimientos de los componentes productivos y los precios de mercado que oscilan de una temporada a otra como lo mencionan (Collaguazo, 2019)\& (Coronel, 2019).

\section{Conclusiones.}

- Los pobladores de la parroquia rural de Flores identificaron con los valores más altos los servicios de abastecimientos con un puntaje de 8,5/10 siendo el mejor valorado el aprovisionamiento de alimentos, los servicios con menor valoración fueron los servicios culturales con $6 / 10$ de los cuales el menor puntuado fue el de rituales ancestrales. La variable sociodemográfica de la edad incide en la valoración de los servicios de abastecimiento y culturales.

- El 15\% de la población está dispuesta a pagar por la conservación del recurso vegetal productivo con un DAP estimado de $\$ 10,20$ el mayor número de encuestados el $85 \%$ no están dispuestos a pagar y la principal razón para no pagar que expresaron fue que la responsabilidad de la conservación es del gobierno.

- El valor económico total calculado fue de \$724.730,63 de los cuales el que mayor aporta es la producción de pasto con $\$ 466.407,50$, seguido por bosques con $\$ 201.268,51$, DAP con $\$ 34.873,8$ y finalmente el que menos aporta es la producción 
agrícola con $\$ 22.189,82$, esto debido a que esta es un producción marginal y pobre con poca inversión y deficiencia de riego.

\section{Referencias bibliográficas}

Agbenyega et al. (2019). Application of an Ecosystem Function Framework to Perceptions of Community Woodlands. Researchgate, 1, 4.

Briceño, J. I.-G. (2016). Factores que influyen en la percepción de servicios de los bosques secos en el sur del Ecuador. Ecología, 25(2), 46 - 58. doi:10.7818

Collaguazo, C. (2019). VALORACIÓN ECONÓMICA AMBIENTAL DEL RECURSO VEGETAL PRODUCTIVO DE LA PARROQUIA SAN LUIS, CANTÓN RIOBAMBA, PROVINCIA DE CHIMBORAZO. Trabajo de titulación presentado para optar al grado académico de Ingeniera en Biotecnología Ambiental, Riobamba.

Consejo Cantonal de Protección de derechos GADM Riobamba. (2019). Linea Base de grupos de atención prioritaria del cantón Riobamba. Riobamba.

Coronel, N. (2019). VALORACIÓN ECONÓMICA AMBIENTAL DEL RÍO CHAMBO EN EL TRAMO DEL RELLENO SANITARIO PORLÓN. Trabajo de titulación presentado para optar al grado académico de Ingeniera en Biotecnolgía Ambiental, Riobamba.

Estruch, V. V. (2018). An An economic valuation of ecosystem services provided by the River Turia Natural Park (Valencia). Economía Agraria y Recursos Naturales, $18(2)$.

FAO-Bolivia. (05 de 2013). Saberes ancestrales e indicadores naturales para la reducción de riesgos a desastres agropecuarios. Obtenido de http://www.fao.org/3/a-as976s.pdf

GADPR- Flores. (03 de Abril de 2015). Plan de Desarrollo y Ordenamiento Territorial 2015-2019. Recuperado el 24 de Junio de 2019, de GADPR-Flores: http://app.sni.gob.ec/snilink/sni/PORTAL_SNI/data_sigad_plus/sigadplusdocumentofinal/06608203200 01_PDyOT\%20FLORES\%202015OK_30-10-2015_11-45-

Garcés, N. (2021). Valoración económica ambiental del recurso vegetal de la parroquia Flores, cantón Riobamba. Proyecto de titulación: trabajo previo a la obtención del titulo de Ingeniera en Biotecnología Ambiental, Riobamba.

Guevara, E. (2020). Valoración económica ambiental del recurso suelo en la parroquia rural de Licto. Trabajo de titulación presentado para optar al grado académico de Ingeniero en Biotecnología Ambiental, Escuela Superior Politécnica de Chimborazo. 
Hufnagel, et al. (19 de Septiembre de 2018). Evaluation Methods of Ecosystem Services and Their Scientific and Societal Importance in Service of Solving the Global Problems of the Humankind, Ecosystem Services and Global Ecology, Levente Hufnagel, IntechOpen,. Recuperado el 04 de Diciembre de 2019, de https://www.intechopen.com/books/ecosystem-services-and-globalecology/introductory-chapter-evaluation-methods-of-ecosystem-services-andtheir-scientific-and-societal-impo

INTA. (25 de Septiembre de 2015). Instituto Nacional de Tecnología Agropecuaria. Recuperado el 01 de Mayo de 2020, de https://inta.gob.ar/noticias/serviciosecosistemicos-culturales

Leiva, M. e. (05 de Diciembre de 2018). ResearchGate. Obtenido de LOS SERVICIOS ECOSISTÉMICOS CULTURALES: https://www.researchgate.net/publication/333649430_Capitulo_14_Los_servicio s_ecosistemicos_culturales

Lemache, K. (2020). Valoración economica ambiental del recurso vegetal de la parroquia Punin del cantón Riobamba. Trabajo de titulación previo a la obtención del titulo de Ingeniero en Biotecnología Ambiental, Riobamba. Obtenido de http://dspace.espoch.edu.ec/bitstream/123456789/14524/1/236T0515.pdf

Ministerio del Ambiente-Ecuador. (27 de Abril de 2016). "Estrategia Nacional de Biodiversidad 2015-2030. Recuperado el 21 de Febrero de 2020, de Ministerio del

Ambiente-Ecuador: http://maetransparente.ambiente.gob.ec/documentacion/WebAPs/Estrategia\%20 Nacional\%20de\%20Biodiversidad\%202015-2030\%20\%20CALIDAD\%20WEB.pdf

Paltan, R. (2020). Valoración económica ambiental del recurso suelo en la parroquia rural Pungalá. Trabajo de titulación presentado para optar al grado académico de Ingeniero en Biotecnología Ambiental, Riobamba. Obtenido de http://dspace.espoch.edu.ec/handle/123456789/14269

Villamagua, G. (2017). Percepción social de los servicios ecosistémicos en la microcuenca El Padmi Ecuador. Revista Iberoamericana de Economía Ecológica, 27, 102 -114. doi:ISSN 13902776

VIU. (21 de Marzo de 2018). Universidad Internacional de Valencia. Recuperado el 24 de Abril de 2020, de La productividad marginal y los factores productivos: https://www.universidadviu.com/la-productividad-marginal-y-los-factoresproductivos/

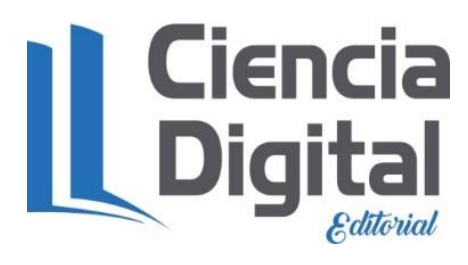


PARA CITAR EL ARTÍCULO INDEXADO.

Brito Mancero, M. Y., \& Garcés Chérres, N. A. (2021). Estimación de los servicios ecosistémicos del recurso vegetal de la parroquia Flores, cantón Riobamba . ConcienciaDigital, $4(3.1)$,

105-121. https://doi.org/10.33262/concienciadigital.v4i3.1.1817

\section{¿Ciencia}

El artículo que se publica es de exclusiva responsabilidad de los autores y no necesariamente reflejan el pensamiento de la Revista Conciencia Digital.

El artículo queda en propiedad de la revista y, por tanto, su publicación parcial y/o total en otro medio tiene que ser autorizado por el director de la Revista Conciencia Digital.

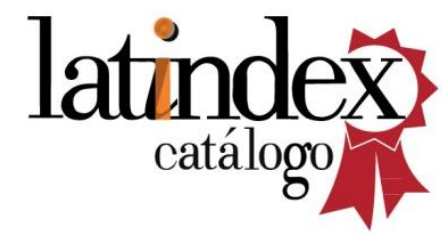

